ARTICLE

\title{
Nanoscale electric-field imaging based on a quantum sensor and its charge-state control under ambient condition
}

\author{
Ke Bian 1,2,3,8, Wentian Zheng (10 1,8, Xianzhe Zeng ${ }^{1}$, Xiakun Chen ${ }^{1}$, Rainer Stöhr ${ }^{2,3}$, Andrej Denisenko ${ }^{2,3}$, \\ Sen Yang $\mathbb{1}^{4}$, Jörg Wrachtrup ${ }^{2,3 凶} \&$ Ying Jiang $\mathbb{Q}^{1,5,6,7 凶}$
}

Nitrogen-vacancy (NV) centers in diamond can be used as quantum sensors to image the magnetic field with nanoscale resolution. However, nanoscale electric-field mapping has not been achieved so far because of the relatively weak coupling strength between NV and electric field. Here, using individual shallow NVs, we quantitatively image electric field contours from a sharp tip of a qPlus-based atomic force microscope (AFM), and achieve a spatial resolution of $\sim 10 \mathrm{~nm}$. Through such local electric fields, we demonstrated electric control of NV's charge state with sub-5 nm precision. This work represents the first step towards nanoscale scanning electrometry based on a single quantum sensor and may open up the possibility of quantitatively mapping local charge, electric polarization, and dielectric response in a broad spectrum of functional materials at nanoscale.

\footnotetext{
${ }^{1}$ International Center for Quantum Materials, School of Physics, Peking University, Beijing, China. ${ }^{2}$ 3rd Institute of Physics, University of Stuttgart and Institute for Quantum Science and Technology (IQST), Stuttgart, Germany. ${ }^{3}$ Max Planck Institute for Solid State Research, Stuttgart, Germany. ${ }^{4}$ Department of Physics, The Chinese University of Hong Kong, Shatin, New Territories, Hong Kong, China. ${ }^{5}$ Collaborative Innovation Center of Quantum Matter, Beijing, China. ${ }^{6}$ CAS Center for Excellence in Topological Quantum Computation, University of Chinese Academy of Sciences, Beijing, China.

7 Interdisciplinary Institute of Light-Element Quantum Materials and Research Center for Light-Element Advanced Materials, Peking University, Beijing, China.

${ }^{8}$ These authors contributed equally: Ke Bian, Wentian Zheng. ${ }^{凶}$ email: j.wrachtrup@pi3.uni-stuttgart.de; yjiang@pku.edu.cn
} 
$\mathrm{T}$ he nitrogen-vacancy (NV) center, an atomic defect in diamond, is one of the most promising candidates for quantum computing ${ }^{1,2}$, quantum information ${ }^{3}$ and quantum sensing $\mathrm{g}^{4,5}$ under ambient condition, relying on the coherent manipulation of the spin $(S=1)$ in its negatively charged state $\left(\mathrm{NV}^{-}\right)$. Previous efforts have proven the shallow NVs to be powerful quantum sensors for detecting external signals, such as magnetic ${ }^{6-9}$, electric fields ${ }^{10-12}$ strain $^{8}$, and temperature ${ }^{13,14}$. In particular, nanoscale magnetic-field imaging is possible by integrating a nano-diamond tip into the atomic force microscopy $(\mathrm{AFM})^{15}$, benefiting from the atomic size of NV. Recently, NVbased scanning magnetometry ${ }^{16,17}$ based on the continuous wave-optical detected magnetic resonance (cw-ODMR) has been successfully used for the quantitative real-space imaging of superconducting vortex ${ }^{18,19}$, multiferroic non-colinear order $^{20}$, skyrmion structure ${ }^{21}$, and $2 \mathrm{D}$ magnetic materials ${ }^{22}$.

In contrast, the electric-field sensing by the NV based on the Stark effect is much more difficult, and NV-based scanning electrometry has not been realized so far. The main reason arises from the relatively weak coupling strength of the $\mathrm{NV}$ with electric field, leading to small energy shifts of spin levels (typically $<0.1$ $\mathrm{MHz})^{10,12}$. In this case, more advanced sensing schemes rather than the simple $\mathrm{cw}$-ODMR are required, which strongly rely on the coherent properties of $\mathrm{NVs}^{23}$. However, the oscillating cantilevers of most non-contact AFM can modulate the distance between NV and target charged elements. Such a modulation induces electric noise, which would decrease the coherence time of the NV and degrades its sensitivity during the electric-field imaging ${ }^{17}$. Thus, it is ideal to bring the source of the external electric field as close as possible to the sensor while maintaining small oscillation amplitudes, which is challenging for the conventional NV-compatible $\mathrm{AFM}^{18-22}$.

In this work, we circumvented this problem by using a qPlusbased $\mathrm{AFM}^{24}$, which allows positioning a conductive tip close to the diamond surface below $1 \mathrm{~nm}$ with very small oscillation amplitudes (typically 100-300 pm). Thanks to the high field gradient of the sharp tip, we realized nanoscale quantitative imaging of external electric-field contours within the sensing regime of this atomic-size electrometer using pulsed-ODMR ${ }^{25}$. Furthermore, with the assistance of such high field gradients and photon ionization by the excitation laser, the local electric field of the tip can be applied to achieve control on the local electrostatic environment and charge state of shallow NVs with nanoscale accuracy (down to $4.6 \mathrm{~nm}$ ), which may substantially enhance the coherence of NV and thus improve its field sensitivity.

\section{Results}

Quantitative electric-field imaging at nanoscale. The experimental setup is schematically shown in Fig. 1a (for details, see the "Methods" section). Shallow NVs with the depth of 5-10 nm were used as quantum probes, whose spin states were initialized by green laser and readout through red fluorescence. As a consequence of ion implantation for NV creation with subsequent annealing and acid-boiling cycles, a large number of defects were introduced both near the NVs and on the diamond surface 26,27 .

\section{a}

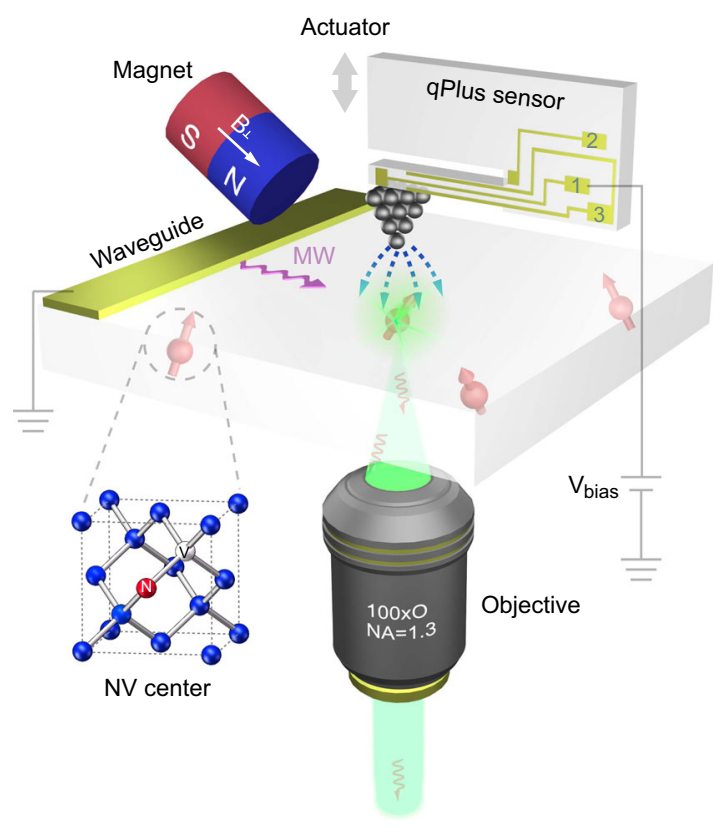

b

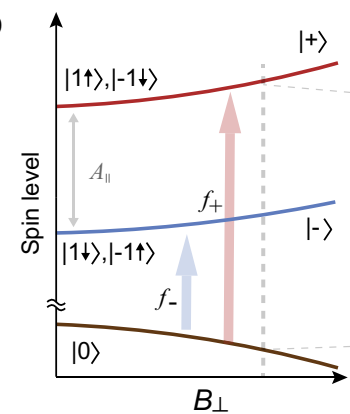

C

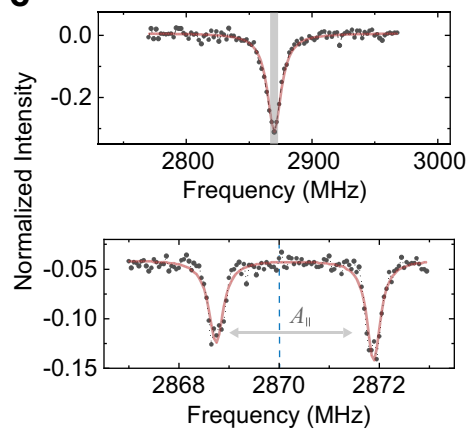

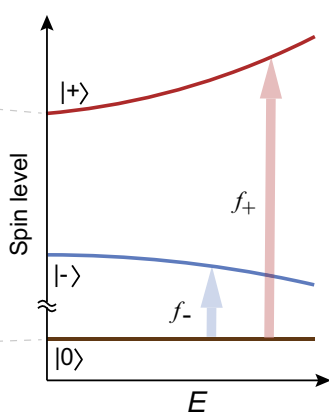

d

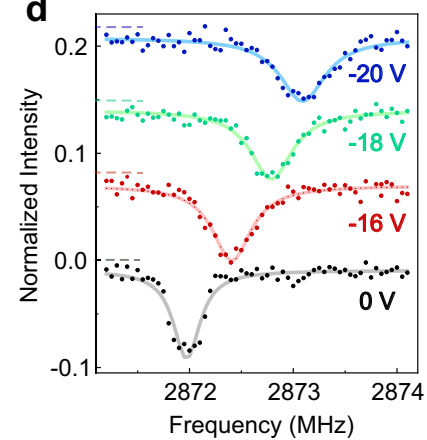

Fig. 1 Sensing the external field from AFM tip using single shallow NV. a Schematic graph of the home-built AFM system combined with NV technology. A metal tip was guided close to a specific NV using a qPlus sensor, where the electrode for bias is denoted by 1 and the electrodes for AFM signals are denoted by 2 and 3. A piezo actuator (gray arrow) is used for exciting the oscillations of qPlus. Microwave (MW) pulses feed through the on-chip waveguide. The transverse magnetic field $B_{\perp}$ is dentoed by a white arrow, with the magnitude of $9.6 \mathrm{G}$. $\mathbf{b}$ Left: The spin levels of NV- under transverse magnetic field with varying strength. Hyperfine interaction of associated $N^{15}$ isotope is denoted by $A_{\|}$. The states $(|1 \uparrow\rangle,|-1 \downarrow\rangle)$ and $(|-1 \uparrow\rangle,|+1 \downarrow\rangle)$ (denoted by $z$-projection of the electron spin-1 and its associated nuclear spin-1/2) are equally mixed into $|+\rangle$ and $|-\rangle$, respectively. Right: Energy levels of $| \pm\rangle$ states under the electric field with varying strength. Arrows indicate the spin resonant transition under the microwaves ( $f_{+}$and $\left.f_{-}\right)$. c Continuous wave-ODMR (upper panel) and pulsed-ODMR (lower panel) under zero electric fields. The hyperfine splitting cannot be resolved in continuous waveODMR (the gray shadow), but is clearly visible in pulsed-ODMR. The vertical dashed line denotes $2.87 \mathrm{GHz}$. $\mathbf{d}$ Pulsed-ODMR spectra showing the electron-spin-resonance shift of $f_{+}$under different tip biases. Curves are offset for clarity. AFM setpoint: $\triangle f_{\text {AFM }}=+20 \mathrm{~Hz}$. 
a

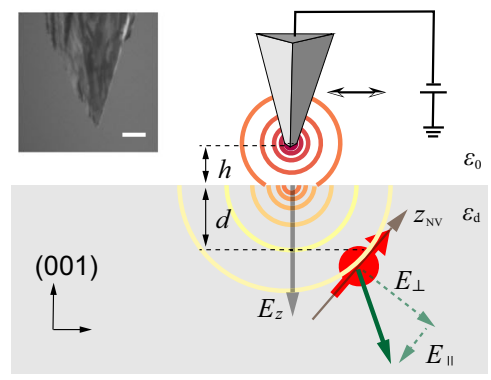

d

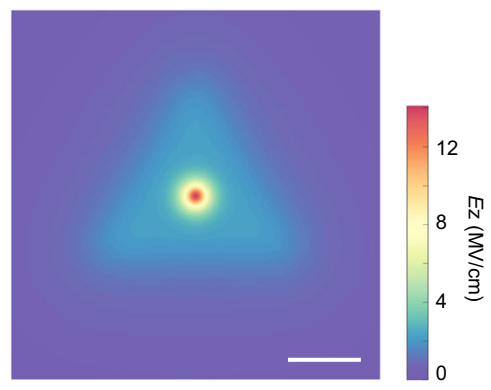

b

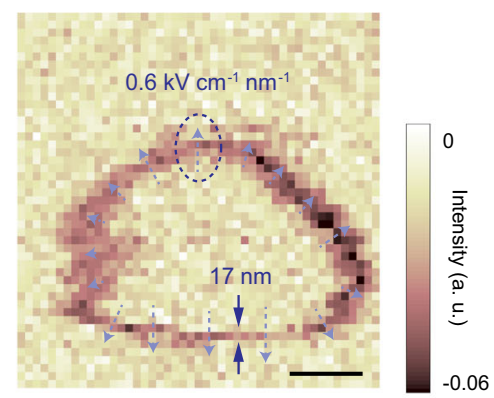

e

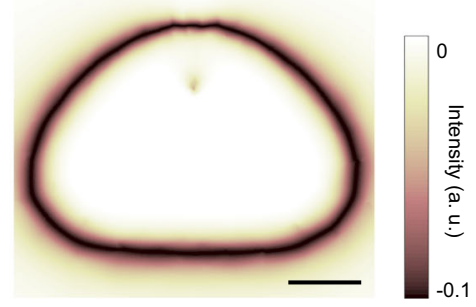

C

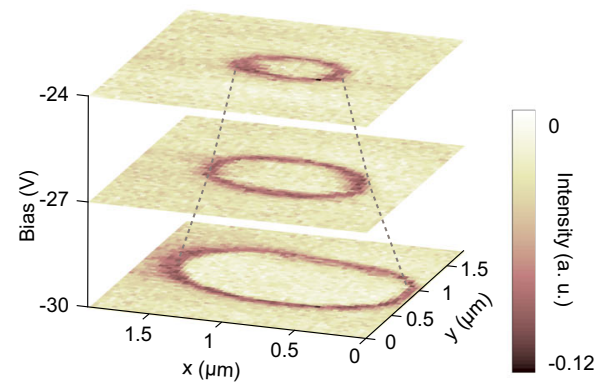

Fig. 2 NV-based nanoscale scanning electrometry and quantitative estimation. a Schematic graph showing the simple triangular-tip model with a spherical tip apex. $E_{\perp}$ and $E_{\|}$(green lines) are projected onto a NV with a depth of $d$ at a tip height of $h$. During the simulation, we set $\varepsilon_{\mathrm{d}}=5.7$ for diamond material. Inset: Scanning electron microscope (SEM) image of FIB-treated tungsten tip. Scale bar: $1 \mu \mathrm{m}$. b Scanning field-gradient imaging obtained at -16 V with ODMR setpoint of $700 \mathrm{kHz}$. The dashed arrows reflect the direction and magnitude of field gradient. c Scanning field-gradient imaging of the tip under different biases. AFM setpoint: $\triangle f_{\mathrm{AFM}}=+10 \mathrm{~Hz}$. Pulsed-ODMR setpoint: $\triangle f=400 \mathrm{kHz}$. d Simulated field distribution along the surface normal ( $E_{z}$ ) from the triangular-tip model (a) with a side length of $220 \mathrm{~nm}$, apex radius of $30 \mathrm{~nm}, h=1 \mathrm{~nm}, d=5 \mathrm{~nm}$ and bias $=-150 \mathrm{~V}$. A field strength as large as $14.1 \mathrm{MV} \mathrm{cm}-1$ can be experienced by the NV. e Simulated scanning-field gradient image at the bias $=-10 \mathrm{~V}$. The ODMR setpoint is set to be $700 \mathrm{kHz}$. The non-spherical shape arises from both the triangular tip and the fact that NV axis is not perpendicular to diamond surface. Scale bars in $\mathbf{b}$, $\mathbf{d}$, and $\mathbf{e}$ are 100 nm. $\mathbf{f} A$ series of simulated scanning gradient-field images under the same geometric parameters as in (e), demonstrating a precision of 13.9 nm for positioning the $\mathrm{NV}$ underneath.

Such defects can act as donors or acceptors, which are also considered to be the major source for decoherence and charge instability of shallow $\mathrm{NVs}^{28,29}$. In order to guide the conductive AFM tip towards a target fluorescent spot with high precision and to maintain a small tip-surface distance for achieving strong electric field, we chose the qPlus sensor working at the frequency modulation (FM) mode 24 . Because of its high stiffness and special asymmetric configuration, the qPlus sensor can work at very small tip heights $(<1 \mathrm{~nm})$ with tiny oscillation amplitudes $(<100$ $\mathrm{pm})$ even under ambient conditions ${ }^{30}$, thus hardly inducing electric noise to affect the coherence of $\mathrm{NV}^{-}$when the tip is biased.

We carefully aligned the magnetic field $(\sim 9.6 \mathrm{G})$ perpendicular to the NV axis according to the hyperfine resonance spectra ${ }^{10}$, such that the eigenstates of this spin-1 system changes into an equal mixture of $m_{\mathrm{s}}= \pm 1$ spin projection, i.e. $| \pm\rangle$ states (Fig. 1b). The strength of electric field can be measured from the frequency shift of $| \pm\rangle$ states due to the Stark effect ${ }^{31}$. Based on the pulsedODMR scheme (Fig. 1c) (for details, see the "Methods" section and Supplementary Fig. 1$)^{25}$, such a frequency shift $(\triangle f)$ is clearly visible under different tip biases (Fig. 1d).

Next, we fixed the microwave frequency to be slightly offresonant and monitored the fluorescence intensity during scanning the biased tip, allowing a single $\Delta f$ to be probed (Fig. 2a). In the scanning field-gradient image with the tip biased at $-16 \mathrm{~V}$, a triangle-like shape of field contour appears (Fig. 2b), which is consistent with the triangular-pyramid tip apex cut by the focused ion beam (FIB) (see inset in Fig. 2a). However, at higher bias voltage and lower $\Delta f$, the electric field imaging changed into a nearly spherical structure (Fig. 2c), reflecting the overall symmetric shape of the tip shaft. From the preset microwave frequency, we can quantitatively estimate the field strength of the projected transverse component $E_{\perp}$ to be $119 \pm$ $3.3 \mathrm{kV} \mathrm{cm}^{-1}$ at the ring position in Fig. 2b (for details of such quantitative estimation, see Supplementary Note 1). The corresponding electric-field gradient can be obtained from the line width of the ring structure and the minimum detectable field strength of the $\mathrm{NV}\left(\sim 17.6 \mathrm{kV} \mathrm{cm}^{-1}\right)$ (see the arrows in Fig. 2b). Figure $2 \mathrm{~d}$ shows the simulated field distribution based on the model of tip with triangular shape (Fig. 2a). By adjusting the geometric parameters of the tip, we obtained the simulated fieldgradient imaging (Fig. 2e), which agrees well with the experimental data (Fig. 2b).

From the field gradient in Fig. 2b, we can estimate that a tip amplitude of several $\mathrm{nm}$ will lead to a spectral broadening comparable to the intrinsic width of the pulsed-ODMR spectra (Supplementary Note 2). Therefore, the small amplitude $(<100$ $\mathrm{pm}$ ) of our qPlus sensor is important to reduce the electric noise. It is worth noting that the best field resolution achieved in this work $(\sim 17 \mathrm{~nm})$ (Fig. $2 \mathrm{~b})$ is determined not only by the absolute field gradient of the tip, but also the field sensitivity and size of the quantum sensor. Such a spatial resolution allows us to locate the AFM tip upon the NVs with high precision (Fig. 2f). We expect a resolution improvement by at least one order of 
a
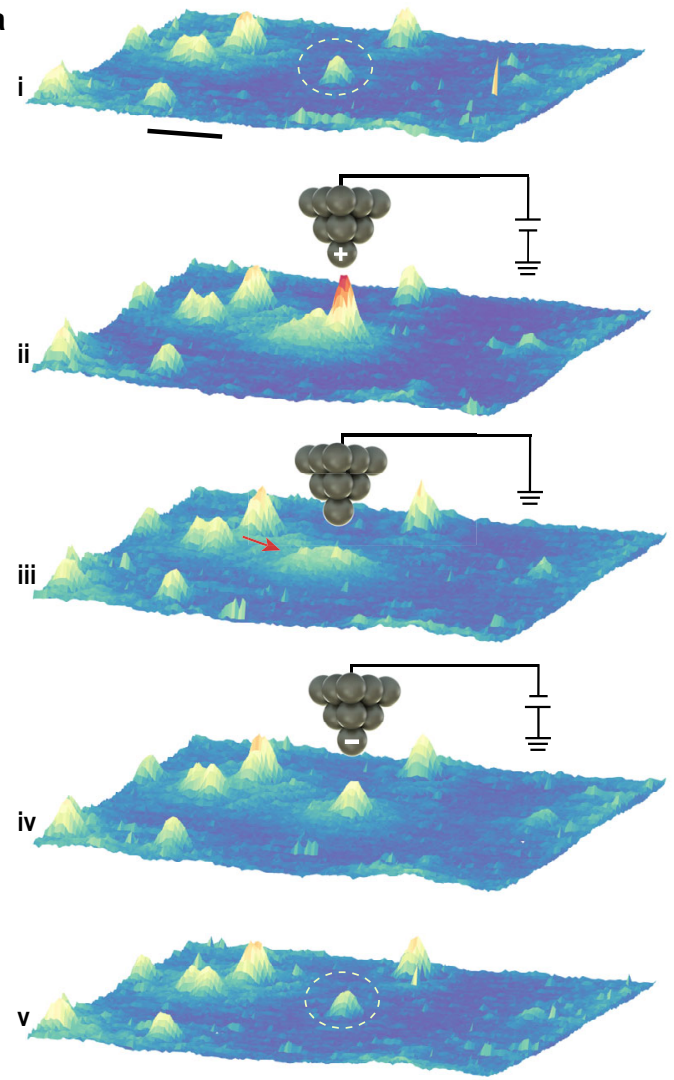

b

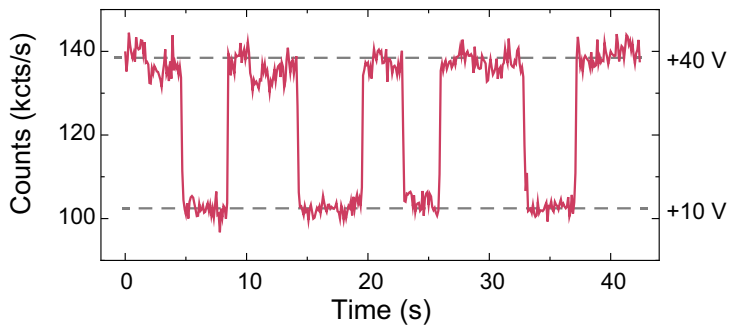

C

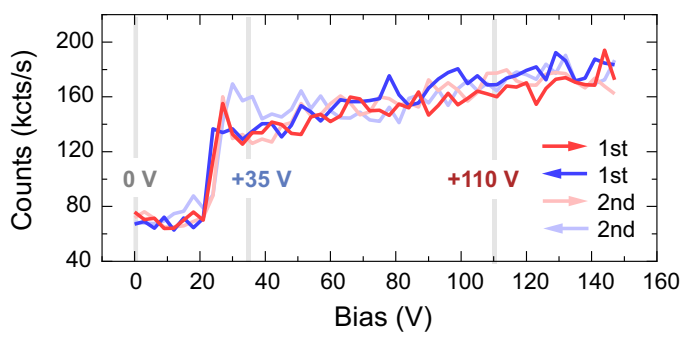

d

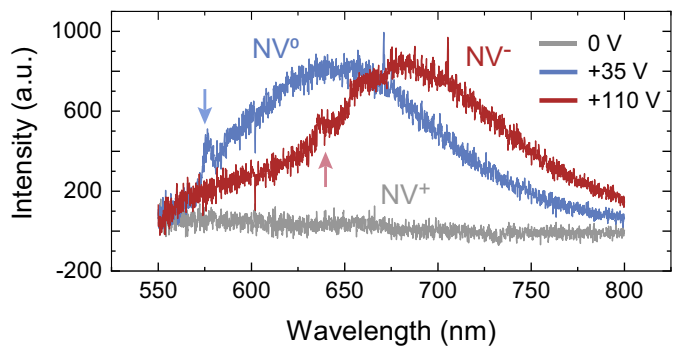

Fig. 3 Tip-induced charge-state transition of a single NV. a The process of charging the NV by local electric field from the AFM tip. Scale bar: $1 \mu \mathrm{m}$. (i) Confocal image including the target NV denoted by a dashed white ellipse before the charge state control. (ii) The tip with a high positive bias was guided close to the target NV, and the fluorescence of NV preserved. (iii) When the tip was grounded, the NV became completely dark. The small background denoted by red arrow arises from the tip-scattered light. (iv) Applying a high negative voltage to the tip switched the NV to bright state as before. (v) The bright state of NV remains when the tip was grounded and retracted. $\mathbf{b}$ Time-elapsed fluorescence shows the fast switch of NV charge state when the bias was set at +40 or $+10 \mathrm{~V}$ alternatively. c Fluorescence-bias curves in the positive voltage range. Reproducible curves along with two cycles of bias ramps indicate the charge state control with high fidelity. $\mathbf{d}$ PL spectra of the same NV in (c) under different biases, confirming the different charge states of NV. The blue and red arrows indicate the $\mathrm{ZPL}$ of $\mathrm{NV}^{0}$ and $\mathrm{NV}^{-}$, respectively. All the fluorescence data were recorded under the 488-nm excitation laser with the 550-nm LP filter.

magnitude if a spin-echo scheme with a synchronized AC bias voltage is applied ${ }^{10,12}$. Finally, using the geometric parameters obtained from the simulation, we deduce that the largest field reachable in our system is $\sim 14 \mathrm{MV} \mathrm{cm}^{-1}$ (Fig. 2d).

Highly efficient charge-state control of NV by AFM tip. In contrast to the magnetic field imaging, measuring of charges and electric fields by NV centers can have a massive effect on the electronic structure of the NV center itself. In particular, the strong electric fields from the sharp tip can result in locally tuning the charge state of single NVs (see more details in Supplementary Fig. 2 and Supplementary Note 3). As shown in Fig. 3a, a positively biased tip $(+90 \mathrm{~V})$ was first positioned above a bright $\mathrm{NV}$, and its fluorescence was slightly enhanced. However, when the tip bias was decreased to zero, this NV became non-luminescent. Its fluorescence revived again after applying a negative tip bias $(-90$ $\mathrm{V}$ ) and remained stable afterwards at zero bias. Figure $3 \mathrm{~b}$ shows the fast switching between the bright and dark states of the NV, in prompt response to the positive bias voltages applied $(+10$ and $+40 \mathrm{~V})$. When ramping the bias voltage on the dark NV, a sharp transition threshold in the fluorescence counts was observed around $+25 \mathrm{~V}$ (Fig. 3c). Such a transition is highly repeatable during multiple cycles of the bias ramp.
The change of the fluorescence state most likely arises from the charge-state transitions of $\mathrm{NVs}^{29}$. To confirm this conclusion, we measured the photoluminescence (PL) of single NVs under 488$\mathrm{nm}$ excitation laser to avoid the spectral overlap between background Raman peak and PL features (Supplementary Fig. 3). Under three selected positive biases, both the characteristic zero phonon line (ZPL) and sideband unambiguously point to the distinct charge states (Fig. 3d). The dark state at zero bias is attributed to $\mathrm{NV}^{+}$state $\mathrm{s}^{32,33}$, while the bright states at +35 and $+100 \mathrm{~V}$ are attributed to $\mathrm{NV}^{0}$ and $\mathrm{NV}^{-}$states ${ }^{34}$, respectively. We note that the transition between $\mathrm{NV}^{0}$ and $\mathrm{NV}^{-}$is more obvious at smaller laser powers (see Supplementary Fig. 4 and Supplementary Note 4). Strikingly, the $\mathrm{NV}^{+}$state remains stable in the absence of the external tip field, even under long-term laser illumination (Supplementary Fig. 5), which is in clear contrast to the previous work ${ }^{32,33}$.

The mechanism of charge-state control. In order to gain deeper insights into the charge transition process, we systematically ramped the tip bias within a larger range (Fig. 4a). From the start, a high positive voltage $(+150 \mathrm{~V})$ was applied on a bright $\mathrm{NV}$ $(\mathrm{NV}-)$ and ramped towards the negative voltage (blue curve in Fig. 4a). The fluorescence suddenly drops around $+25 \mathrm{~V}$ (similar 
a

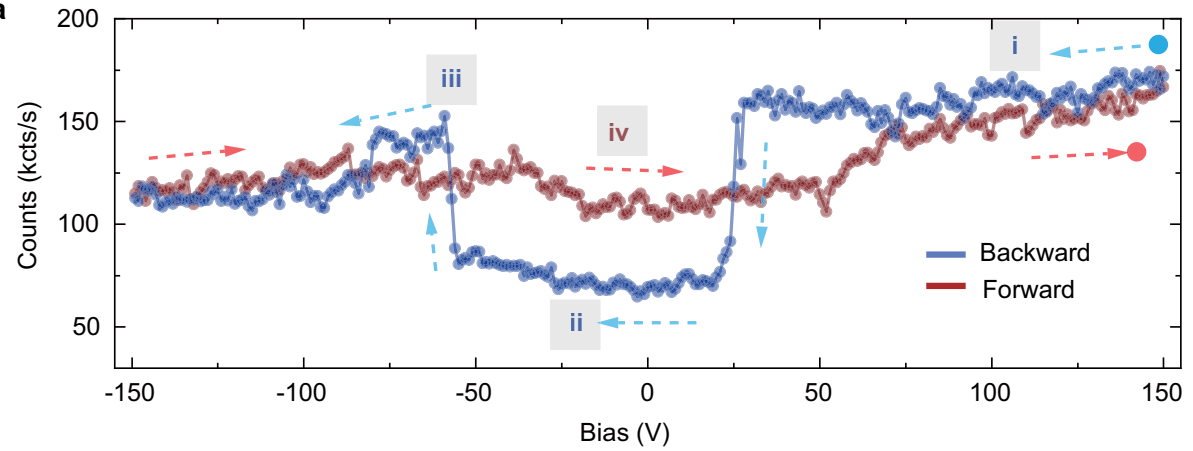

b

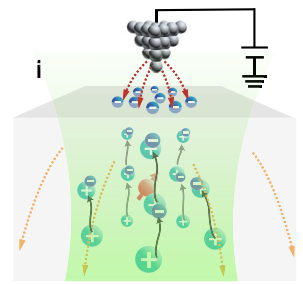

$\mathrm{NV}^{-}$

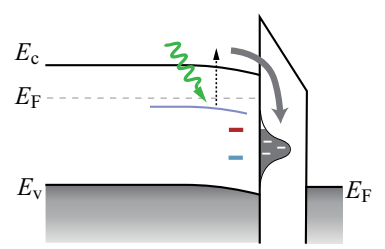

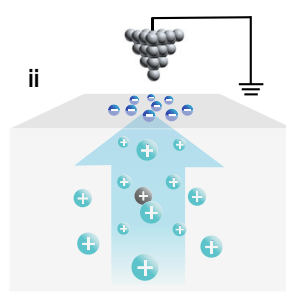

$\mathrm{NV}^{+}$

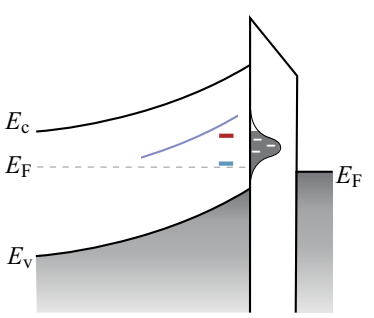

C
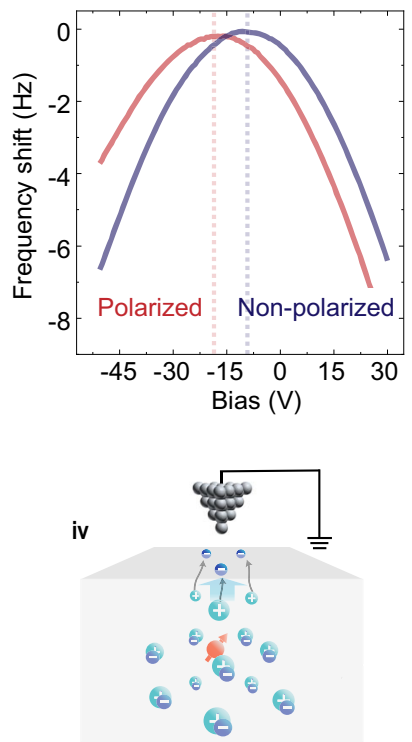

$\mathrm{NV}^{-}$

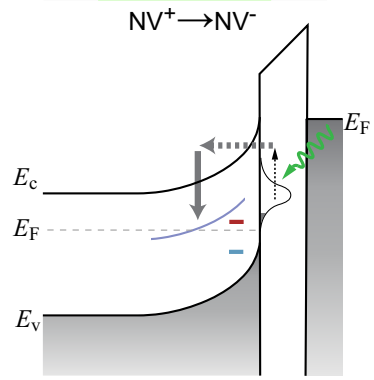

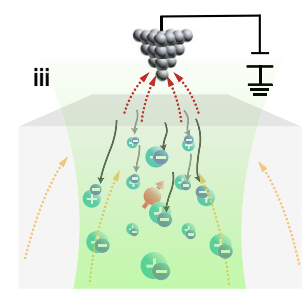

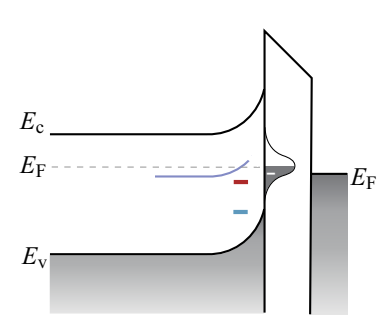

Fig. 4 Schematic model of the charge state control. a Fluorescence-bias curves over the full-bias range. The dashed arrows denote the direction of bias ramp. b Cartoon graphs (upper panels) and energy band diagrams (lower panels) showing the concerted effect of photon ionization and tip electric field. Upper panels: red and black balls indicate NV-and $\mathrm{NV}^{+}$states, respectively. Dashed arrows denote the local electric field. Lower panels: the purple curve denotes the donor's charge transition level, while blue and red short lines denote $\mathrm{NV}^{+} / \mathrm{NV}^{0}$ and $\mathrm{NV}^{0} / \mathrm{NV}^{-}$transition levels, respectively. The waved green arrow denotes exciting photons. $E_{\mathrm{F}}, E_{\mathrm{C}}$, and $E_{\mathrm{V}}$ denote the Fermi level, conduction, and valence band edges of diamond, respectively. (i) Under a large positive bias, lots of photon-ionized electrons (dark blue balls) from the donors (cyan balls) are driven upward and trapped by the surface defects. (ii) A strong built-in electric field (light blue arrow) remains when the tip is grounded, leading to the upward band bending and the NV+ state. (iii) The surfacetrapped electrons are photoexcited and tunnel to the conduction band under negative bias, thence recombined with the donors, depolarizing the surface and leading to the $\mathrm{NV}^{-}$state again. (iv) When the bias is grounded again, only shallow donors are depleted due to the electron transfer to surface defects and a small built-in electric field is formed, thus the $\mathrm{NV}^{-}$state remains. $\mathbf{c}$ LCPD measurements confirmed the negatively charged surface after polarization. Setpoint: $\Delta f_{\text {AFM }}=+20 \mathrm{~Hz}$.

to Fig. 2c) and recovers around $-50 \mathrm{~V}$, which correspond to the $\mathrm{NV}^{0} \rightarrow \mathrm{NV}^{+}$and $\mathrm{NV}^{+} \rightarrow \mathrm{NV}^{-}$transitions (see Supplementary Fig. $3 \mathrm{~b}$ and c), respectively. Along the opposite ramp (red curve in Fig. 4a), however, the photon counts show negligible variations. We excluded the electron injection and the ionization effect of air as the origin of $\mathrm{NV}$ charging/discharging ${ }^{35-37}$, considering the fact that the $\mathrm{NV}^{+} \rightarrow \mathrm{NV}^{-}$transition occurs at both positive and negative biases, and that the charge state is still tunable even if the tip is lifted several tens of nanometers above the diamond surface.

We recall that the manipulated $\mathrm{NV}^{+}$does not relax back to $\mathrm{NV}^{-}$ in the absence of the external electric field. In addition, the charge state of NV becomes tunable only after applying a sufficiently high positive bias voltage. Therefore, the $\mathrm{NV}^{+}$should be stabilized by the intrinsic surface polarization, which is induced by the strong tip field and preserves after the field is removed. Meanwhile, after turning off the laser, no charge transition was observed on the diamond surface even under large biases $( \pm 120 \mathrm{~V})$. Therefore, we propose a model based on surface electron trapping arising from the concerted effect of photon-ionization and strong local electric fields.
Defects such as substitution nitrogen (P1 center) and vacancy complex act as deep donors and weakly dope the lattices surrounding the implanted NVs ${ }^{27,38}$. Recently, the existence of $s p^{2}$ carbon defects on the diamond surface with a density of $>4 \times$ $10^{13} \mathrm{~cm}^{-2}$ caused by different surface treatments has also been confirmed by XNAES and DFT calculations ${ }^{26}$. As shown in Fig. $4 \mathrm{~b}(\mathrm{i})$, the donors are easily ionized by the excitation of 488 or $532-\mathrm{nm}$ laser due to its proximity to the conduction band minimum $(\sim 1.7 \mathrm{eV})^{38}$. Those ionized electrons are attracted towards the surface by the positively charged tip and trapped in the unsaturated carbon defects at the surface. Considering that those surface defect states appear 3.3-4 eV below the conduction band minimum ${ }^{26}$ and the lack of mobile holes in the valence band, the trapped electrons are very stable under thermal equilibrium condition. Together with the ionized donors underneath, the charged surface establishes a large built-in electric field at zero tip bias ((ii) in Fig. 4b), depleting $\mathrm{NV}^{-}$to $\mathrm{NV}^{+}$. Such a built-in electric field competes with the tip-induced downward band bending under the positive bias ${ }^{39-41}$, hence enabling the charge-state transition as shown in Fig. 3c. When reversing the 


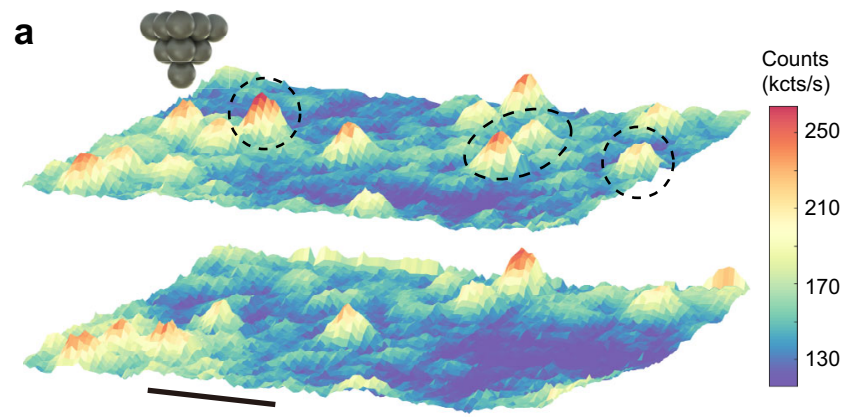

C
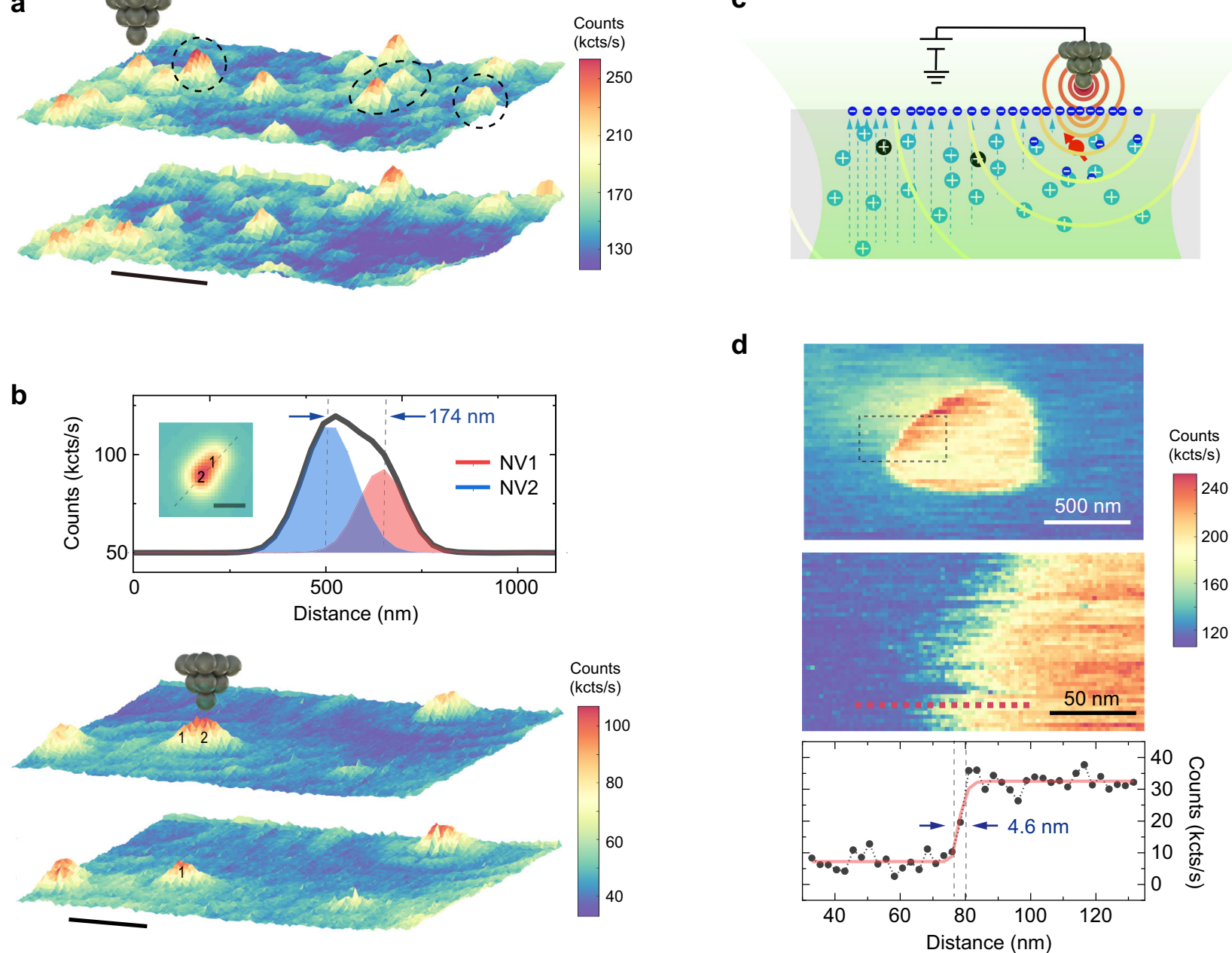

Fig. 5 Local charge-state control with high spatial resolution. a Confocal image showing highly efficient charge-state control of arbitrarily selected NVs. The target NVs are denoted by the black dashed circles. Scale bar: $1 \mu \mathrm{m}$. $\mathbf{b}$ Charge state control of a NV dimer with a separation beyond the diffraction limit. The manipulated NV is denoted by 2. Scale bar: $0.6 \mu \mathrm{m}$. Upper panel: Line profile across the dimer and the corresponding Gaussian fit, yielding a separation distance of $\sim 174 \mathrm{~nm}$. Inset: Simulated confocal image of the NV dimer. Scale bar: $250 \mathrm{~nm}$. c Schematic graph demonstrating the basic principle of chargestate transition imaging. The AFM tip with a high field gradient is able to control the charge state of NV with high spatial resolution. The green shade denotes excitation laser, while the semi-circled curves denote electrostatic potential contour from the conducting tip. The dashed lines indicate the built-in electric field. d Charge-state transition image of a single NV. Upper panel: A disk-like structure was obtained when scanning the metal tip with a high positive bias $(+90 \mathrm{~V})$. The edge of the disk reflects the $\mathrm{NV}^{+} / \mathrm{NV}^{0}$ transition. Middle panel: The zoomed-in image as denoted by the black dashed rectangle in the upper panel. Lower panel: The profile along the red dashed line depicted in the middle panel. After subtracting a linear slope, the line profile was fitted by a step function, yielding a spatial resolution of $4.6 \mathrm{~nm}$ for charge-state control.

bias polarity, those surface-trapped electrons are repelled back into the bulk through photo-assisted tunneling process, and recombine with the ionized donors ((iii) in Fig. 4b). Consequently, the surface is depolarized along with the disappearance of the built-in electric field, leading to the revival of the $\mathrm{NV}^{-}$state ((iv) in Fig. 4b). It is worth noting that we did not obtain $\mathrm{NV}^{+}$up to a large negative bias (Fig. 4a), but only a small component of $\mathrm{NV}^{0}$ mixed into $\mathrm{NV}^{-}$states (Supplementary Fig. 3c). This might arise from the less efficient screening of the negatively charged tip than that of the positive tip.

The built-in electric field induced by surface electron trapping can be further confirmed by local contact potential difference (LCPD) measurements ${ }^{42}$ (see Supplementary Note 5 and Fig. 4c). We found that the contact potential of the tip in the polarized region is significantly more negative than that in the nonpolarized region, consistent with that the polarized surface is negatively charged with trapped electrons. We also studied the role of surface chemistry in the charge-state manipulation. The charge states of NVs in two samples with different surface treatments (see the "Methods" section and Supplementary Fig. 6) are both tunable, except for a significantly larger positive threshold bias on the cleaner one (see Supplementary Fig. 7 and Supplementary Note 6). We conclude that the surface adsorbates can saturate the surface defects through charge transfer, leading to less efficient electron trapping and thus the decreased built-in electric field.

Spatial precision of local charge-state control. Finally, we demonstrate spatial control on the charge state of single NVs with high precision. In Fig. 5a, the randomly distributed bright $\mathrm{NV}^{-}$ can be selectively switched to dark $\mathrm{NV}^{+}$. In Fig. 5b, we chose a $\mathrm{NV}$ dimer with a separation of $\sim 170 \mathrm{~nm}$, which is beyond the resolution of our confocal imaging. The charge state of NVs in this dimer can be well-controlled individually. In order to explore the ultimate limit of spatial control on the charge state, we obtained a charge-state transition image of a single $\mathrm{NV}$ by 
continuously recording fluorescence as scanning a positively biased tip (Fig. 5c). The resulting disk-like structure (upper panel in Fig. 5d) is very similar to the field contour image obtained by pulsed-ODMR (Fig. 2b). The sharp transition edge (middle panel in Fig. $5 \mathrm{~d}$ ), which arises from the $\mathrm{NV}^{+} / \mathrm{NV}^{0}$ transition, yields a spatial resolution of $\sim 4.6 \mathrm{~nm}$ for charge state control (lower panel in Fig. 5d). Such a high spatial resolution is attributed to the high field gradient of the tip apex and the fast charge transfer near NV in response to the external field.

\section{Discussion}

In conclusion, we achieved the nanoscale imaging of electric fields using the NV as a local electrometer under ambient condition. In the future, integrating the $\mathrm{NV}$ onto the scanning tip may become an emerging tool in nanotechnology and opens up the possibility of probing the local charge and electric polarization in a broad spectrum of functional materials, such as solar cells, ion batteries, ferroelectrics, multiferroics, electronic devices, etc. However, the $\mathrm{NV}$ inside the diamond tip after complex fabrication process suffers from low coherence time, low spin contrast and poor charge stability, which limit the sensitivity of diamond tips and its application for electric-field measurement. Recently, the nanometer-sized diamond pillar array with a flat top has been successfully produced and the sensitivity of NV is comparable to ones inside non-structured diamonds. The next step would be transferring and integrating the single diamond nanopillar onto the AFM tip. Besides, it is noteworthy to mention that despite the considerable shielding effect from the diamond host, there is no significant distortion in the scanning electrometry image and degradation of the spatial resolution when the flat-top nanopillar with a $200-\mathrm{nm}$ diameter is used for E-field sensing (Supplementary Note 7 and Supplementary Fig. S8). The absolute strength of the external electric field can be extracted by the careful pre-calibration of the NV-based quantum sensor using well-defined standard samples.

Clearly, the sensitivity of the current sensing technique is not as good as the most sensitive scanning E-field imaging techniques, such as scanning single-electron-transistor (SET) ${ }^{43,44}$ and scanning quantum dot (QD) microscopy ${ }^{45,46}$. However, most of those techniques only work under low temperature or ultrahigh vacuum conditions. Another asset of NV-based scanning electrometry is the potential to quantitatively measure the electric field, which is very challenging for electrostatic force microscopy $(\mathrm{EFM})^{47,48}$ or kelvin probe force microscopy $(\mathrm{KPFM})^{49}$. Furthermore, the nanoscale spatial resolution of the scanning NV electrometry is not significantly influenced by the nanostructured diamond tip such as the flat-top diamond nanopillar mentioned above (Supplementary Fig. S8). Such a feature may be complementary to other scanning E-field probes, whose spatial resolution is more sensitive to the tip apex, such as the size of micro-sensors (scanning SET, scanning QD), the details of the exact tip termination (KPFM, EFM), etc.

Feeding AC electric field to the AFM tip may open up further possibility of exploring frequency-dependent surface dielectric response at nanoscale based on ultrasensitive coherent measurements. By tuning the local electrostatic environment such as appropriately depleting the surrounding charges of $\mathrm{NV}$ in a controllable manner, our technique is promising to reduce the spin/charge noise and enhance the spin coherence/contrast of the shallow $\mathrm{NVs}^{50}$, which can improve the electric-field sensitivity even up to single elementary charge.

In addition, we have also demonstrated that the electric control on the charge-state transition of NV can be realized with sub-5 $\mathrm{nm}$ precision. The manipulated charge states are intrinsically stable under thermal equilibrium condition, which is, in principle, applicable for other kinds of solid-state qubits in diamond ${ }^{51,52}$ or silicon carbide ${ }^{53}$. Those results pave the way to construct complex qubit network for scalable quantum register and quantum processor, especially on samples containing ensemble qubits such as NVs. The strong local field and the small capacitance from the sharp tip can greatly enhance the transfer speed of charge carriers and enable the fast charging/discharging of NV within microseconds, which is vital for nuclear-based quantum storage ${ }^{33}$ and spin to charge readout ${ }^{54,55}$.

\section{Methods}

Experimental setup. All the data in this work were recorded in our home-built scanning probe microscope (SPM) system, which was specially designed for achieving excellent compatibility with NV center technology. The SPM part includes a compact Pan-type scanner head ${ }^{56}$, integrated with vector magnets and high-frequency transmission cables. An oil-immersed objective $(\mathrm{NA}=1.3)$ was used for photon collection, and the focus spot was driven by a commercial piezoelectric scanner (Physik Instrumente). We chose two types of qPlus sensors (one with $k=1.8 \mathrm{kN} \mathrm{m}^{-1}, Q \sim 2000, f=33 \mathrm{kHz}$, the other with $k=3.6 \mathrm{kN} \mathrm{m}^{-1}, Q \sim 2500$, $f=53 \mathrm{kHz})$ equipped with a tungsten tip $(25 \mu \mathrm{m}$ in diameter) for AFM measurements ${ }^{15}$. The tungsten tip was first electrochemically etched in the $\mathrm{NaOH}$ solution, followed by cleaning and sharpening with FIB. The bias voltage was supplied by an AO output of NI-DAQ (National Instrument) and amplified through a commercial voltage amplifier $(-150$ to $+150 \mathrm{~V}$, CoreMorrow). The bias was applied on the tip, and the ground reference was on the waveguide and shielding box of the SPM scanner. The details of the NV-combined SPM will be published elsewhere. Single NV centers were located by our home-built confocal imaging system. The spin state of $\mathrm{NV}^{-}$was initialized by a $532-\mathrm{nm}$ laser and readout by fluorescence lights. The 532-nm laser was chopped by an AcousticOptic modulator (AOM, Gooch \& Housego) in the double-pass working mode and subsequently shaped by a single-mode fiber. A half-wave plate was used for adjusting the polarization of input laser. Fluorescence photons through 650-nm long-pass (LP) filter were ultimately collected by avalanche photodiode, excelitas (APD). A 100- $\mathrm{mm}$ multi-mode fiber was connected to APD and used as the pinhole in our confocal setup. We used the NI-DAQ for counting photons. Microwaves (MW) were generated by a Keysight signal generator (N5181B), chopped by switchers, amplified by a Minicircuit power amplifier, and then fed through onchip waveguide for flipping the electron spin of $\mathrm{NV}^{-}$. The synchronization of laser pulse, microwave pulse and counter timing was executed through a multi-channeldigital pulse generator (PBESR-500, Spincore). The AFM data acquisition was realized by a commercial Nanonis package, while the photon counts and coherent manipulation were realized by the self-programmed Labview VIs. PL spectra were recorded by a spectrometer from Princeton Instrument (SP2300). When performing PL measurements, the 532-nm laser was replaced by a 488-nm one and the $650-\mathrm{nm}$ LP was replaced by a $550-\mathrm{nm}$ one to avoid the spectral overlap between background Raman peak and PL features of $\mathrm{NV}^{0}$. The typical acquisition time of PL spectra is 2-30 min per curve depending on the signal-to-noise ratio under excitation laser of $400 \sim 500 \mu \mathrm{W}$, during which the charge state of NV was not changed. The obtained PL spectra of NV was then normalized by a reference PL spectral acquired on the diamond region without NV.

Pulsed-ODMR and nanoscale electric-field imaging. For pulsed-ODMR measurements, we applied a microwave $\pi$-pulse for flipping the electron spin (Supplementary Fig. 1a), during which no laser was illuminated on the NV. In this case, the detection sensitivity of external electric field is largely enhanced compared to continuous-wave ODMR (Supplementary Fig. 1b), because of the suppressed power-broadening induced by continuous excitation laser and microwave. The spectral resolution depends on the duration time of $\pi$-pulse (Supplementary Fig. 1c), which is ultimately limited by the NV's coherence time ${ }^{25}$. In our experiments, the maximum duration of $\pi$-pulse is $\sim 5.4 \mu \mathrm{s}$, leading to a spectral resolution of $\sim 300 \mathrm{kHz}$, which corresponds to the minimum detectable field strength of $\sim 17.6 \mathrm{kV} \mathrm{cm}^{-1}$ and sensitivity of $\sim 35.2 \mathrm{kV} \mathrm{cm}^{-1} \mathrm{~Hz}^{-1 / 2}$ (Supplementary Fig. 1d). For scanning field-gradient imaging 57 , we fixed the frequency of microwave pulse several hundreds of $\mathrm{kHz}$ away from the resonance peak under the zero field. The resulting frequency shift reflects the strength of electric field, under which the spin resonance decreased photon counts. The tip-surface distance was controlled through oscillation amplitudes $(100-300 \mathrm{pm})$ and frequency shifts $(+10$ to $+70 \mathrm{~Hz}$ ) of the qPlus sensor in frequency-modulation (FM) mode. The integration time per pixel is $2-4 \mathrm{~s}$, leading to a total acquisition time of $2-4 \mathrm{~h}$ per image (48 pixels $\times 48$ pixels). All the fluorescence data for electric field sensing were recorded under the 532-nm excitation laser with the 650-nm LP filter. All the pulsed-ODMR data were normalized by $f_{\text {sig }} / f_{\text {ref }}$, where $f_{\text {sig }}$ and $f_{\text {ref }}$ are the averaged fluorescence photons within $\sim 300$-ns signal and reference countingwindow defined in Supplementary Fig. 1d. A temperature control system was built for suppressing the thermal drift ${ }^{58}$. The temperature setpoint was maintained by 4 PID (proportional-integrating-differential) units, and the whole NV-SPM system was settled inside a thermal insulation box consisting of foams and acoustic-proof panels. 
NV creation and sample treatment. The diamonds are commercial electronicgrade single-crystal chips purchased from Element Six. The intrinsic nitrogen concentration is below $5 \mathrm{ppb}$. The chips were milled into membranes with a thickness of $20-30 \mu \mathrm{m}$ by laser cutting in DDK Inc. The diamond membranes were then implanted with $5-\mathrm{keV} \mathrm{N}^{15}$ ions. A subsequent high-temperature annealing led to the diffusion of carbon vacancies, which were ultimately combined with $\mathrm{N}^{15}$ donors. For sample A, in order to avoid complex process in ultraviolet (UV) lithography or electron beam lithography (EBL), we chose the etched copper films as a shadow mask during the evaporation of $\mathrm{Cr} / \mathrm{Au}$ waveguide (Supplementary Fig. 6a). Because of the $\sim 1-\mathrm{mm}$ distance between the mask and diamond substrate, a small amount of $\mathrm{Cr} / \mathrm{Au}$ was leaked into the waveguide gaps, leading to high confocal background (100-120 kcts s ${ }^{-1}$ under $\left.300 \mu \mathrm{W}\right)$ and quenching of some NVs. After putting the chip into piranha solution and boiling it for three cycles, the confocal background was decreased to $10-20 \mathrm{kcts} \mathrm{s}^{-1}$ under $300 \mu \mathrm{W}$ and more NVs revived (Supplementary Fig. 6b). The AFM image shows that the surface of sample A is covered by 5 -nm-thick layers, which may correspond to the adsorbed water under ambient condition due to the hydrophilic termination of the surface after the acid treatment. For sample B, after the NV creation the chip was immersed in isopropanol solution for several months (Supplementary Fig. 6c). To keep the sample surface relatively clean, we did not evaporate any waveguide and perform acid-boiling procedure on sample B. From the AFM image, the surface of sample B is free of the adsorbed layers as found on sample A (Supplementary Fig. $6 \mathrm{~d}$ ). Both the A and B chips were stuck onto a $170-\mu \mathrm{m}$ silica substrate using UV-cured glues and inserted into our SPM system. The typical saturated fluorescence of single NVs in our system is $70-90 \mathrm{kcts} \mathrm{s}^{-1}$ with a background of 10-15 $\mathrm{kcts} \mathrm{s}^{-1}$. The spin contrast of NVs on sample A measured through Rabi oscillation is $15-25 \%$, and the typical $\mathrm{T}_{2}$ under spin-echo sequence is $15-30 \mu$ s.

\section{Data availability}

The data that support the findings of this study are available from the corresponding author upon reasonable request.

Received: 28 July 2020; Accepted: 24 March 2021; Published online: 28 April 2021

\section{References}

1. Jelezko, F. et al. Observation of coherent oscillation of a single nuclear spin and realization of a two-qubit conditional quantum gate. Phys. Rev. Lett. 93, 130501 (2004).

2. Waldherr, G. et al. Quantum error correction in a solid-state hybrid spin register. Nature 506, 204-207 (2014).

3. Bernien, $H$. et al. Heralded entanglement between solid-state qubits separated by three metres. Nature 497, 86-90 (2013).

4. Boss, J. M., Cujia, K. S., Zopes, J. \& Degen, C. L. Quantum sensing with arbitrary frequency resolution. Science 356, 837-840 (2017).

5. Schmitt, S. et al. Submillihertz magnetic spectroscopy performed with a nanoscale quantum sensor. Science 356, 832-836 (2017).

6. Shi, F. Z. et al. Single-protein spin resonance spectroscopy under ambient conditions. Science 347, 1135-1138 (2015).

7. Aslam, N. et al. Nanoscale nuclear magnetic resonance with chemical resolution. Science 357, 67-71 (2017)

8. Hsieh, S. et al. Imaging stress and magnetism at high pressures using a nanoscale quantum sensor. Science 366, 1349-1354 (2019).

9. Yip, K. Y. et al. Measuring magnetic field texture in correlated electron systems under extreme conditions. Science 366, 1355-1359 (2019).

10. Dolde, F. et al. Electric-field sensing using single diamond spins. Nat. Phys. 7, 459-463 (2011).

11. Broadway, D. A. et al. Spatial mapping of band bending in semiconductor devices using in situ quantum sensors. Nat. Electron. 1, 502-507 (2018).

12. Michl, J. et al. Robust and accurate electric field sensing with solid state spin ensembles. Nano Lett. 19, 4904-4910 (2019).

13. Kucsko, G. et al. Nanometre-scale thermometry in a living cell. Nature 500, 54-58 (2013).

14. Neumann, P. et al. High-precision nanoscale temperature sensing using single defects in diamond. Nano Lett. 13, 2738-2742 (2013).

15. Giessibl, F. J. Advances in atomic force microscopy. Rev. Mod. Phys. 75, 949-983 (2003).

16. Maletinsky, P. et al. A robust scanning diamond sensor for nanoscale imaging with single nitrogen-vacancy centres. Nat. Nanotechnol. 7, 320-324 (2012).

17. Balasubramanian, G. et al. Nanoscale imaging magnetometry with diamond spins under ambient conditions. Nature 455, 648-651 (2008)

18. Pelliccione, M. et al. Scanned probe imaging of nanoscale magnetism at cryogenic temperatures with a single-spin quantum sensor. Nat. Nanotechnol. 11, 700-705 (2016).
19. Thiel, L. et al. Quantitative nanoscale vortex imaging using a cryogenic quantum magnetometer. Nat. Nanotechnol. 11, 677-681 (2016).

20. Gross, I. et al. Real-space imaging of non-collinear antiferromagnetic order with a single-spin magnetometer. Nature 549, 252-256 (2017).

21. Dovzhenko, Y. et al. Magnetostatic twists in room-temperature skyrmions explored by nitrogen-vacancy center spin texture reconstruction. Nat. Commun. 9, 2712 (2018).

22. Thiel, L. et al. Probing magnetism in $2 \mathrm{D}$ materials at the nanoscale with single-spin microscopy. Science 364, 973-976 (2019).

23. Taylor, J. M. et al. High-sensitivity diamond magnetometer with nanoscale resolution. Nat. Phys. 4, 810-816 (2008).

24. Giessibl, F. J. The qPlus sensor, a powerful core for the atomic force microscope. Rev. Sci. Instrum. 90, 011101 (2019).

25. Dreau, A. et al. Avoiding power broadening in optically detected magnetic resonance of single NV defects for enhanced dc magnetic field sensitivity. Phys. Rev. B 84, 195204 (2011).

26. Stacey, A. et al. Evidence for primal $s p(2)$ defects at the diamond surface: candidates for electron trapping and noise sources. Adv. Mater. Interfaces 6, 1801449 (2019).

27. de Oliveira, F. F. et al. Tailoring spin defects in diamond by lattice charging. Nat. Commun. 8, 15409 (2017).

28. Hauf, M. V. et al. Chemical control of the charge state of nitrogen-vacancy centers in diamond. Phys. Rev. B 83, 081304 (2011).

29. Bluvstein, D., Zhang, Z. R. \& Jayich, A. C. B. Identifying and mitigating charge instabilities in shallow diamond nitrogen-vacancy centers. Phys. Rev. Lett. 122, 076101 (2019).

30. Purckhauer, K. et al. Imaging in biologically-relevant environments with AFM using stiff qPlus sensors. Sci. Rep. 8, 9330 (2018).

31. Doherty, M. W. et al. Theory of the ground-state spin of the $\mathrm{NV}^{-}$center in diamond. Phys. Rev. B 85, 205203 (2012)

32. Hauf, M. V. et al. Addressing single nitrogen-vacancy centers in diamond with transparent in-plane gate structures. Nano Lett. 14, 2359-2364 (2014).

33. Pfender, M. et al. Protecting a diamond quantum memory by charge state control. Nano Lett. 17, 5931-5937 (2017).

34. Aslam, N., Waldherr, G., Neumann, P., Jelezko, F. \& Wrachtrup, J. Photoinduced ionization dynamics of the nitrogen vacancy defect in diamond investigated by single-shot charge state detection. New J. Phys. 15, 013064 (2013).

35. Repp, J., Meyer, G., Olsson, F. E. \& Persson, M. Controlling the charge state of individual gold adatoms. Science 305, 493-495 (2004).

36. Fatayer, S. et al. Molecular structure elucidation with charge-state control. Science 365, 142-145 (2019).

37. Patera, L. L., Queck, F., Scheuerer, P. \& Repp, J. Mapping orbital changes upon electron transfer with tunnelling microscopy on insulators. Nature 566, 245-248 (2019).

38. Deak, P., Aradi, B., Kaviani, M., Frauenheim, T. \& Gali, A. Formation of NV centers in diamond: a theoretical study based on calculated transitions and migration of nitrogen and vacancy related defects. Phys. Rev. B 89, 079905 (2014).

39. Teichmann, K. et al. Controlled charge switching on a single donor with a scanning tunneling microscope. Phys. Rev. Lett. 101, 076103 (2008).

40. Lee, D. H. \& Gupta, J. A. Tunable field control over the binding energy of single dopants by a charged vacancy in GaAs. Science 330, 1807-1810 (2010).

41. Zheng, H., Weismann, A. \& Berndt, R. Tuning the electron transport at single donors in zinc oxide with a scanning tunnelling microscope. Nat. Commun. $\mathbf{5}$, 2992 (2014)

42. Melitz, W., Shen, J., Kummel, A. C. \& Lee, S. Kelvin probe force microscopy and its application. Surf. Sci. Rep. 66, 1-27 (2011).

43. Yoo, M. J. et al. Scanning single-electron transistor microscopy: Imaging individual charges. Science 276, 579-582 (1997).

44. Martin, J. et al. Observation of electron-hole puddles in graphene using a scanning single-electron transistor. Nat. Phys. 4, 144-148 (2008).

45. Wagner, C. et al. Scanning quantum dot microscopy. Phys. Rev. Lett. 115 , 026101 (2015)

46. Cadeddu, D. et al. Electric-field sensing with a scanning fiber-coupled quantum dot. Phys. Rev. Appl. 8, 031002 (2017).

47. Martin, Y., Abraham, D. W. \& Wickramasinghe, H. K. High-resolution capacitance measurement and potentiometry by force microscopy. Appl. Phys. Lett. 52, 1103-1105 (1988).

48. Gross, L. et al. Measuring the charge state of an adatom with noncontact atomic force microscopy. Science 324, 1428-1431 (2009).

49. Henning, A. K. et al. 2-Dimensional surface dopant profiling in silicon using scanning Kelvin probe microscopy. J. Appl. Phys. 77, 1888-1896 (1995).

50. Anderson, C. P. et al. Electrical and optical control of single spins integrated in scalable semiconductor devices. Science 366, 1225-1230 (2019).

51. Sipahigil, A. et al. Indistinguishable photons from separated silicon-vacancy centers in diamond. Phys. Rev. Lett. 113, 113602 (2014). 
52. Evans, R. E. et al. Photon-mediated interactions between quantum emitters in a diamond nanocavity. Science 362, 662-665 (2018).

53. Koehl, W. F., Buckley, B. B., Heremans, F. J., Calusine, G. \& Awschalom, D. D. Room temperature coherent control of defect spin qubits in silicon carbide. Nature 479, 84-87 (2011).

54. Shields, B. J., Unterreithmeier, Q. P., de Leon, N. P., Park, H. \& Lukin, M. D. Efficient readout of a single spin state in diamond via spin-to-charge conversion. Phys. Rev. Lett. 114, 136402 (2015).

55. Siyushev, P. et al. Photoelectrical imaging and coherent spin-state readout of single nitrogen-vacancy centers in diamond. Science 363, 728-731 (2019).

56. Pan, S. H., Hudson, E. W. \& Davis, J. C. He-3 refrigerator based very low temperature scanning tunneling microscope. Rev. Sci. Instrum. 70, 1459-1463 (1999).

57. Grinolds, M. S. et al. Quantum control of proximal spins using nanoscale magnetic resonance imaging. Nat. Phys. 7, 687-692 (2011).

58. Haberle, T. et al. Nuclear quantum-assisted magnetometer. Rev. Sci. Instrum. 88, 013702 (2017).

\section{Acknowledgements}

The authors thank Xinyu Pan and Gangqin Liu for the generous help on NV instrumentations, Zhihai Cheng for valuable suggestions on AFM technologies, Nan Zhao and Marcus Doherty for insightful discussions on the data interpretation, and Bowen Song for valuable assistance on graphics and data processing. This work was financially supported by the National Key R\&D Program under Grant Nos. 2016YFA0300901, 2017YFA0205003, the National Natural Science Foundation of China under Grant Nos. 11888101, 11634001, 21725302, the Strategic Priority Research Program of Chinese Academy of Sciences under Grant No. XDB28000000 and Beijing Municipal Science \& Technology Commission under Grant No. Z181100004218006. R.S., A.D., and J.W. were supported by the ERC grant SMeL and VW Foundation as well as the EU via the projects ASTERIQS and QIA. S.Y. was supported by Hong Kong RGC (GRF/ECS/24304617, GRF/14304618, and GRF/14304419).

\section{Author contributions}

Y.J. and J.W. designed and supervised the project. K.B. and Y.J. constructed the experimental setup. R.S. and A.D. grew diamond chips and fabricated shallow NVs. K.B. and W.Z. performed experiments and data acquisition. X.Z. performed finite-element analysis. K.B., W.Z., X.Z., X.C., S.Y., J.W., and Y.J. performed data analysis and interpretation. K.B. and Y.J. wrote the manuscript with the inputs from all other authors. All the authors commented on the final manuscript.

\section{Competing interests}

The authors declare no competing interests.

\section{Additional information}

Supplementary information The online version contains supplementary material available at https://doi.org/10.1038/s41467-021-22709-9.

Correspondence and requests for materials should be addressed to J.W. or Y.J.

Peer review information Nature Communications thanks Fazhan Shi, Jean-Philippe Tetienne and the other, anonymous, reviewer for their contribution to the peer review of this work

Reprints and permission information is available at http://www.nature.com/reprints

Publisher's note Springer Nature remains neutral with regard to jurisdictional claims in published maps and institutional affiliations.

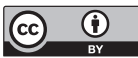

Open Access This article is licensed under a Creative Commons Attribution 4.0 International License, which permits use, sharing, adaptation, distribution and reproduction in any medium or format, as long as you give appropriate credit to the original author(s) and the source, provide a link to the Creative Commons license, and indicate if changes were made. The images or other third party material in this article are included in the article's Creative Commons license, unless indicated otherwise in a credit line to the material. If material is not included in the article's Creative Commons license and your intended use is not permitted by statutory regulation or exceeds the permitted use, you will need to obtain permission directly from the copyright holder. To view a copy of this license, visit http://creativecommons.org/ licenses/by/4.0/.

(C) The Author(s) 2021 